\title{
The Basic Helix-Loop-Helix Transcription Factor SmbHLH1 Represses Anthocyanin Biosynthesis in Eggplant
}

\begin{abstract}
Zhaofei Duan ${ }^{1 \dagger}$, Shiyu Tian ${ }^{1 \dagger}$, Guobin Yang ${ }^{1}$, Min Wei ${ }^{1,2,3}$, Jing Li $^{1,3,4 *}$ and Fengjuan Yang ${ }^{1,3,4 *}$
'State Key Laboratory of Crop Biology, College of Horticulture Science and Engineering, Shandong Agricultural University, Shandong, China, ${ }^{2}$ Scientific Observing and Experimental Station of Facility Agricultural Engineering (Huang-Huai-Hai Region), Ministry of Agriculture and Rural Affairs, Shandong, China, ${ }^{3}$ Shandong Collaborative Innovation Center for Fruit and Vegetable Production With High Quality and Efficiency, Tai'an, China, ${ }^{4}$ Key Laboratory of Biology and Genetic Improvement of Horticultural Crops in Huanghuai Region, Ministry of Agriculture and Rural Affairs, Shandong, China
\end{abstract}

OPEN ACCESS

Edited by:

Carlos R. Figueroa,

University of Talca, Chile

Reviewed by:

Sitakanta Pattanaik,

University of Kentucky,

United States

Qiaomei Wang,

Zhejiang University, China

*Correspondence:

Jing $L i$

lij19900525@163.com

Fengjuan Yang

beautyyfj@163.com

tThese authors have contributed equally to this work

Specialty section:

This article was submitted to Plant Metabolism and Chemodiversity,

a section of the journal

Frontiers in Plant Science

Received: 13 August 2021 Accepted: 05 October 2021 Published: 12 November 2021

Citation:

Duan Z, Tian S, Yang G, Wei M, Li J and Yang F (2021) The Basic Helix-Loop-Helix Transcription Factor SmbHLH1 Represses Anthocyanin Biosynthesis in Eggplant.

Front. Plant Sci. 12:757936. doi: 10.3389/fpls.2021.757936
Many basic helix-loop-helix transcription factors (TFs) have been reported to promote anthocyanin biosynthesis in numerous plant species, but little is known about bHLH TFs that inhibit anthocyanin accumulation. In this study, SmbHLH1 from Solanum melongena was identified as a negative regulator of anthocyanin biosynthesis. However, SmbHLH1 showed high identity with SmTT8, which acts as a SmMYB113-dependent positive regulator of anthocyanin-biosynthesis in plants. Overexpression of SmbHLH1 in eggplant caused a dramatic decrease in anthocyanin accumulation. Only the amino acid sequences at the $\mathrm{N}$ and $\mathrm{C}$ termini of SmbHLH1 differed from the SmTT8 sequence. Expression analysis revealed that the expression pattern of $S m b H L H 1$ was opposite to that of anthocyanin accumulation. Yeast two-hybrid $(\mathrm{Y} 2 \mathrm{H})$ and bimolecular fluorescence complementation (BiFC) assays showed that SmbHLH1 could not interact with SmMYB113. Dual-luciferase assay demonstrated that $\mathrm{SmbHLH} 1$ directly repressed the expression of SmDFR and SmANS. Our results demonstrate that the biological function of bHLHs in anthocyanin biosynthesis may have evolved and provide new insight into the molecular functions of orthologous genes from different plant species.

Keywords: eggplant, anthocyanin biosynthesis, bHLH transcription factor, negative regulation, RNA-seq

\section{INTRODUCTION}

Anthocyanins are water-soluble pigments that are responsible for the red, purple, and blue colors of different organs in a wide range of plants (Mol et al., 1998; Koes et al., 2005; Allan et al., 2008). In addition to coloring flowers and fruits to attract pollinators and seed dispersers, anthocyanins can also protect plants from UV damage and promote resistance to low-temperature stress in plants (Ilk et al., 2015). Anthocyanins have been found to be the most powerful free radical scavengers in plants, and they have been used to lower blood pressure, improve vision, reduce inflammation, and prevent cancer (Konczak and Zhang, 2004; Tsuda, 2012).

Anthocyanins are an important branch of the flavonoid biosynthetic pathway and are formed in a stepwise series of enzymatic reactions catalyzed by chalcone synthase (CHS), chalcone 
isomerase (CHI), flavanone-3-hydroxylase (F3H), flavonoid $3^{\prime}$-hydroxylase $\left(\mathrm{F} 3^{\prime} \mathrm{H}\right)$, flavonoid $3^{\prime} 5^{\prime}$-hydroxylase $\left(\mathrm{F}^{\prime} 5^{\prime} \mathrm{H}\right)$, dihydroflavonol 4-reductase (DFR), anthocyanidin synthase (ANS), and a 3-glucosyltransferase (3GT; Koes et al., 2005). These anthocyanin biosynthetic genes are transcriptionally regulated by a MYB-bHLH-WD40 (MBW) complex, the crucial role of which has been well-documented across many plants, such as Arabidopsis, apple, tomato, and so on (Xie et al., 2012; Tohge et al., 2017; Sun et al., 2020a). AcMYB123 and AcbHLH42, the Actinidia chinensis cv. Hongyang orthologs of Arabidopsis TT2 (MYB) and TT8 (bHLH), respectively, interact to activate the promoters of AcANS and AcF3GT1 and elevate anthocyanin accumulation in Actinidia arguta cv. Baby star and transgenic Arabidopsis thaliana (Wang et al., 2019). In apple, MdbHLH3 interacts with MdMYB1, MdMYB9, and MdMYB11 to promote anthocyanin or proanthocyanidin (PA) accumulation (Xie et al., 2012; An et al., 2014).

The bHLH proteins are the second largest class of transcription factors (TFs) in plants; they can be divided into 26 subgroups (Pires and Dolan, 2010), and this classification can be extended to 32 subgroups if the "atypical" bHLHs are included (CarreteroPaulet et al., 2010). Compared with the 11-13 subgroups of the R2R3 MYBs (Li et al., 2012; He et al., 2016; Huang et al., 2018), bHLH family seems to have a more complex role in anthocyanin regulation. In general, the first 200 amino acids of the bHLH protein are involved in the interaction with the MYB partner, whereas the following 200 amino acids interact with the WD40 protein (Montefiori et al., 2015).

The G-box (5'-CACGTG-3') or E-box (5'-CANNTG-3') in gene promoter regions is recognized by bHLH family members (Atchley et al., 1999; Montefiori et al., 2015; Wang et al., 2018). Strikingly, the promoters of most flavonoid or anthocyanin biosynthetic genes contain G-box and E-box elements (Massari and Murre, 2000). Therefore, in addition to forming the MBW complex, bHLHs can also directly regulate anthocyanin biosynthesis. For example, MdbHLH3 can activate the expression of $M d M Y B 1, M d M Y B 9$, and $M d M Y B 11$ to promote anthocyanin accumulation (Xie et al., 2012; An et al., 2014). TT8 is known to interact with MYB protein to enhance anthocyanin biosynthesis (Baudry et al., 2006; Li et al., 2016b; Wang et al., 2019). However, TT8 orthologs in different species may also perform different functions. Jia et al. (2021) found that DcTT8 from Dendrobium candidum could directly activate the expression of $D c F 3^{\prime} H$ and DcUFGT by binding to their promoters. Zhai et al. (2020) found that targeted mutations of BnTT8 blocked PA-specific deposition, but elevated seed oil content and altered fatty acid (FA) composition in the seed coat of Brassica napus. In addition, bHLHs can also negatively regulate anthocyanin biosynthesis. To data, some bHLH genes have been reported as repressors of anthocyanin biosynthesis in plants, including IN1 from maize (Burr et al., 1996), bHLH3/13/14/17 from Arabidopsis (Song et al., 2013), LcbHLH92 from Leymus chinensis (Zhao et al., 2018), and CpbHLH1 from Chimonanthus praecox (Zhao et al., 2020), but the molecular mechanisms of their negative regulation have been little reported.

Eggplants (Solanum melongena) with purple peel are rich in anthocyanins and popular with consumers. They rank fourth in facility vegetable cultivation planting area. Noda et al. (2000) reported that eggplant extracts showed the most potent superoxide anion radical scavenging activity (SOD-like activity) of 16 common vegetables examined, highlighting the important role of anthocyanins in eggplant. Recently, research focused on the molecular mechanisms of anthocyanin biosynthesis in eggplant has increased (Jiang et al., 2016; Li et al., 2017, 2018; Moglia et al., 2020; Zhou et al., 2020), but gene functions have been verified primarily by ectopic expression in Arabidopsis or tobacco. The eggplant genome database was updated by Barchi et al. (2019), providing a valuable resource for the molecular breeding of new eggplant germplasm. Based on the updated eggplant genome, Moglia et al. (2020) functionally characterized the genes belonging to the MBW complex (SmelANT1, SmelAN2, SmelJAF13, and SmelAN1), and identified out an R3 MYB type repressor (SmelMYBL1) of anthocyanin biosynthesis.

Based on the two published eggplant genome databases (Hirakawa et al., 2014; Barchi et al., 2019), SmbHLH1 (Sme2.5_00592.1_g00005.1) and SmTT8 (SMEL_009g326640.1) were identified as two TT8 homologs. SmTT8 has been shown to interact with SmMYB75 to promote anthocyanin biosynthesis in eggplant (Shi et al., 2021). However, no studies on SmbHLH1 have been reported. Hence, we overexpressed SmbHLH1 in eggplant, and obtained two stable transformation lines, SmbHLH1-1 and SmbHLH1-2. The anthocyanin content was markedly reduced in both lines, indicating that $S m b H L H 1$ and SmTT8 have different functions and molecular mechanisms in the regulation of anthocyanin biosynthesis in eggplant. We used biochemical experiments and transcriptomic analysis to investigate the molecular mechanism by which SmbHLH1 regulates anthocyanin biosynthesis.

\section{MATERIALS AND METHODS}

\section{Plant Materials and Growth Conditions}

Six eggplant cultivars with different color and anthocyanin contents in the fruit and stem peels, named No. 44, No. 64, No. 76, No. 108, No. 109, and No. 133 (Supplementary Figure S1), were grown in the solar greenhouse of Shandong Agricultural University to perform phenotypic observations and collect experimental materials. No. 109 was used for genetic transformation.

Nicotiana benthamiana was grown in a phytotron under $25 / 16^{\circ} \mathrm{C}$ and $12 / 12 \mathrm{~h}$ day/night conditions. Plants with 5-6 leaves were used for bimolecular fluorescence complementation (BiFC), dual luciferase, and transient expression assays.

\section{Anthocyanins Content Analysis}

The anthocyanin content was measured with the methods described by Neff and Chory (1998).

\section{Gene Isolation and Sequence Analysis}

The full-length coding sequences (CDSs) of SmbHLH1 and SmTT8 were cloned by PCR amplification. The amino acid sequences of SmbHLH1 and SmTT8 were translated with DNAMAN 6.0 
(Lynnon Biosoft, Quebec, Canada) based on the sequencing results. Multiple sequence alignments were performed using ClustalX (version 1.83) and DNAMAN 6.0. The MEGA 7.0 program was used to construct neighbor-joining phylogenetic trees with the following parameters: bootstrapping (1,000 replicates, random seed), Poisson model, and complete deletion (Kumar et al., 2016).

\section{Subcellular Localization}

The full-length CDS of SmbHLH1 without the stop codon was inserted into the pHB:GFP vector. The fusion construct was transferred into Agrobacterium tumefaciens strain GV3101, and subcellular localization assays were performed as previously reported (Sparkes et al., 2006).

\section{Yeast Two-Hybrid Assay}

The full-length CDSs of SmbHLH1 and SmTT8 were inserted into the pGBKT7 vector as bait, and $\operatorname{SmMYB113}$ was fused to the pGADT7 vector as prey. The $\mathrm{Y} 2 \mathrm{H}$ assays were performed as described in Zhou et al. (2020).

\section{Bimolecular Fluorescence Complementation}

The full-length CDSs of SmbHLH1 and SmTT8 without stop codons were inserted into the pXY104 vector, and SmMYB113 with the stop codon was subcloned into the pXY106 vector. BiFC assays were performed as described by $\mathrm{Li}$ et al. (2016a).

\section{Plasmid Construction and Plant Transformation}

The full-length CDS of SmbHLH1 was inserted into the pRI 101 vector with the $35 \mathrm{~S}-\mathrm{CaMV}$ promoter. The fusion vector was transferred into Agrobacterium strain LBA4404 and introduced into No. 109 by Agrobacterium-mediated cotyledon explant transformation based on an earlier report by Vasudevan et al. (2005) with minor modifications.

\section{Dual Luciferase and Transient Expression Assays in $\mathbf{N}$. benthamiana Leaves}

The complete coding regions of $S m M Y B 113, S m b H L H 1$, and SmTT8 were inserted into the $\mathrm{pHB}$ vector, and the promoters of anthocyanin biosynthetic genes (SmCHS, SmCHI, SmF3H, $S m F 3^{\prime} H, S m F 3^{\prime} 5^{\prime} H$, SmDFR, SmANS, and Sm3GT) were amplified and cloned into pGreenII 0800-LUC vectors. The Dual-LUC assay was performed as previously reported (Hu et al., 2019). For the transient expression assays, $1 / 2 \times, 1 \times$, and $2 \times$ pHB-SmbHLH1 was co-infiltrated with pHB-SmMYB113 or pHB-SmMYB113+ pHB-SmTT8. The anthocyanin content was measured 4 days after infiltration with the methods described in $\mathrm{Li}$ et al. (2017).

\section{Quantitative Reverse Transcription PCR}

The fruit and stem peels of six eggplant cultivars and thetender stems close to the growing point of No.109 were collected, and total RNA was extracted from the samples using the
TaKaRa MiniBEST Plant RNA Extraction Kit (TaKaRa, Otsu, Shiga, Japan) according to the manufacturer's instructions. Then $1 \mu \mathrm{g}$ RNA was used for cDNA synthesis with the PrimeScript RT Reagent Kit with gDNA Eraser (TaKaRa). The quantitative rreverse ttranscription PCR (RT-qPCR) assay was performed according to the manufacturer's instructions of the SYBR Premix Ex Taq II Kit (TaKaRa) on the LightCycler 96 system (Roche, Basel, Switzerland). The Actin gene (GU984779.1) was used as an internal control gene. The relative expression levels of the amplified products were analyzed using the comparative CT method (Livak and Schmittgen, 2001).

\section{RNA-Seq and Bioinformatics Analyses}

Tender stems close to the growing point were collected from WT and two SmbHLH1 transgenic eggplants for RNA-seq. RNA extraction, quality control, library construction, and highthroughput parallel sequencing were performed at the Beijing Genomics Institute (BGI) on the BGISEQ-500 sequencing platform. Bioinformatics analyses were performed as previously reported (Li et al., 2019).

\section{Statistical Analysis}

SPSS 17.0 (SPSS, Inc., Chicago, United States) was used to assess statistical significance by one-way ANOVA and Duncan's New Multiple Range test $(p \leq 0.05)$.

\section{RESULTS}

\section{Molecular Cloning and Characterization of TT8 Homologs in Eggplant}

The CDSs of SmbHLH1 and SmTT8 were amplified based on the two published eggplant genome databases (Hirakawa et al., 2014; Barchi et al., 2019). After sequencing and alignment, we found that the SmTT8 sequence showed some differences from the published sequence (SMEL_009g326640.1), whereas SmbHLH1 was identical to Sme2.5_00592.1_g00005.1 (Supplementary Figure S2). We then aligned the amino acid sequences of SmbHLH1 and SmTT8 (Figure 1) and found that only the regions at the $\mathrm{N}$ terminus (amino acids $1-158$ and 188-193) and $\mathrm{C}$ terminus (amino acids 633-659) differed between SmbHLH1 and SmTT8.

A phylogenetic tree was constructed to analyze the relationships of SmbHLH1 and SmTT8 with all bHLH proteins from Arabidopsis, Solanum lycopersicum, Malus $\times$ domestica, Vitis vinifera, Fragaria $\times$ ananassa, Petunia $\times$ hybrida, Prunus persica, Chimonanthus praecox, and Medicago truncatula known to be involved in the anthocyanin biosynthesis pathway (Supplementary Figure S3; Li et al., 2017; Zhao et al., 2020). As shown in Supplementary Figure S3, SmbHLH1 and SmTT8 were placed in the same clade.

\section{Different Expression Pattern of SmbHLH1 and SmTT8 in Eggplant}

To analyze the relationship between SmbHLH1 and SmTT8, the expression pattern of SmbHLH1 and SmTT8 were measured 


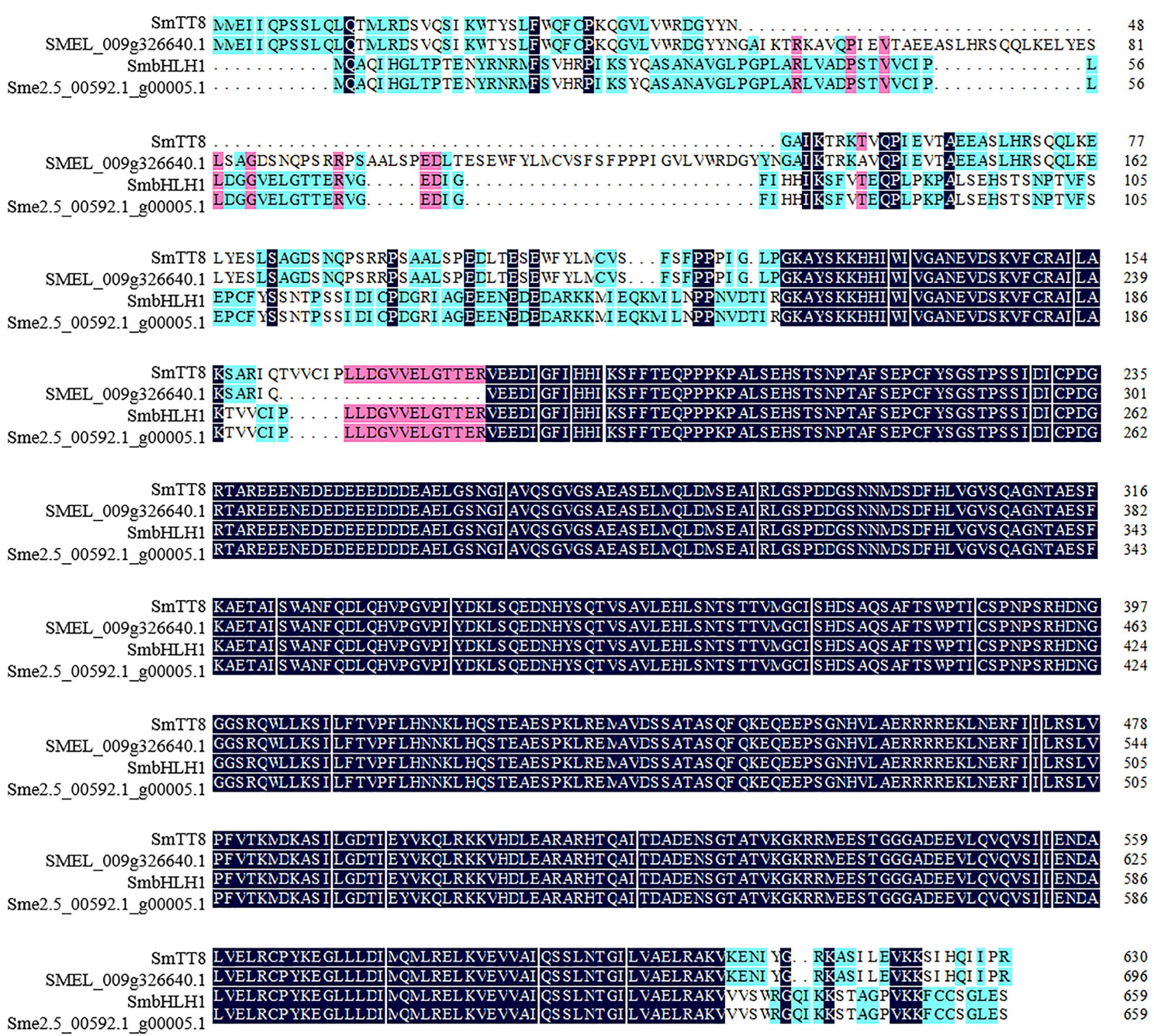

FIGURE 1 | Multiple sequence alignments of the amino acids of SmbHLH1 and SmTT8. SMEL_009g326640.1 and Sme2.5_00592.1_g00005.1 were both annotated as TT8 in the two published eggplant genome databases.

in the fruit and stem peels from six eggplant cultivars with different anthocyanin contents (Figure 2A; Supplementary Figure S1). The primers for RT-qPCR were designed from the $5^{\prime}$ termini, which differed between $S m b H L H 1$ and SmTT8 (Supplementary Table S1). As shown in Figure 2A and Supplementary Figure S1, the anthocyanin content in the fruit peel was sorted as No. $76>$ No. $64>$ No. $44>$ No. $108 /$ No. $109 /$ No. 133 , while No. $76 /$ No. $109>$ No. $44>$ No. $64>$ No. 108/No. 133 in the stem peel. The expression patterns of SmTT8 in the fruit and stem peel were both consistent with anthocyanin contents (Figure 2B). However, the expression pattern of SmbHLH1 was opposite with anthocyanin contents in both fruit and stem peel (Figure 2C). These results implied that the function of SmbHLH1 may be different from that of SmTT8.

To confirm the subcellular location of $S m b H L H 1$, the 35S::SmbHLH1-GFP fusion protein was constructed and transiently expressed in tobacco leaves. Compared with the positive control, SmbHLH1-GFP was predominately present in the cytosol and nucleus (Figure 2D).

\section{Overexpression of SmbHLH1 Inhibited Anthocyanin Accumulation in Eggplant}

To characterize the function of $S m b H L H 1$ in eggplant, transient VIGS assay, CRISPR-Cas9 system, and overexpression system were all performed on all the six eggplant cultivars. However, the approaches to transform the eggplant with the transient VIGS assay and CRISPR-Cas9 system were failed. So we decided to transform the eggplant with $35 \mathrm{~S}$ promoter-constructs to probe the overall function of the SmbHLH1 gene. SmbHLH1 was introduced into eggplant by Agrobacterium-mediated transformation. Only the eggplant cultivar No.109 differentiated two SmbHLH1 overexpression transgenic lines (overexpressed by the 35S-CaMV promoter, named as SmbHLH1-1 and SmbHLH1-2), which would be used to probe the overall function of the SmbHLH1. Both of the two 35S::SmbHLH1 transgenic eggplant lines showed increased SmbHLH1 expression (Figures 3A,B). As shown in Figure 3A, the whole plant of the two $35 \mathrm{~S}:: S m b H L H 1$ transgenic eggplant lines were greener than WT. Especially, the colors of stalks, sepals, and stem 
A

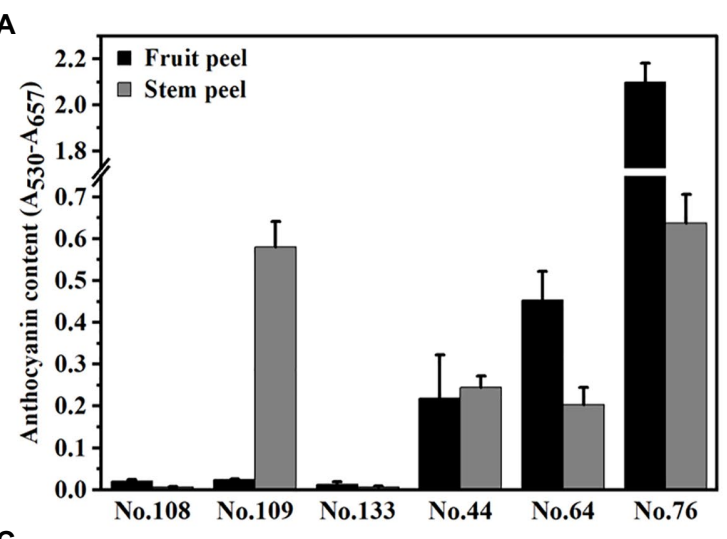

C

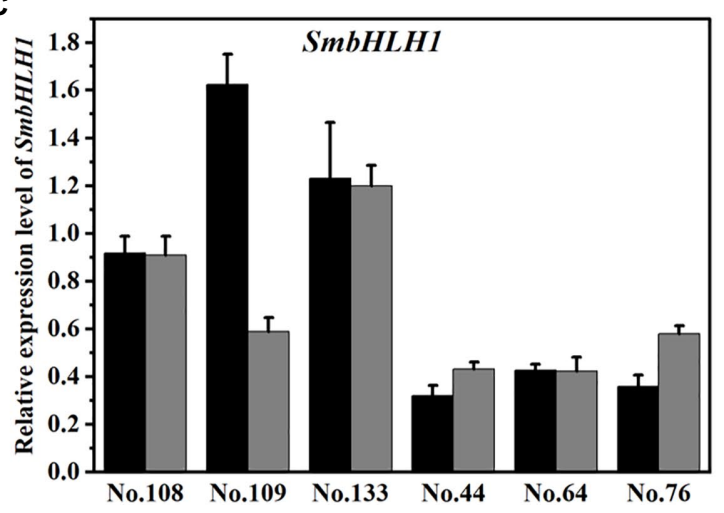

B

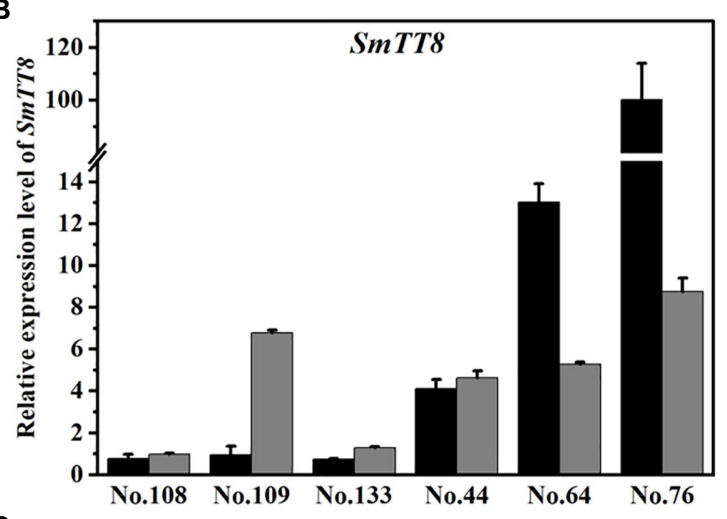

D

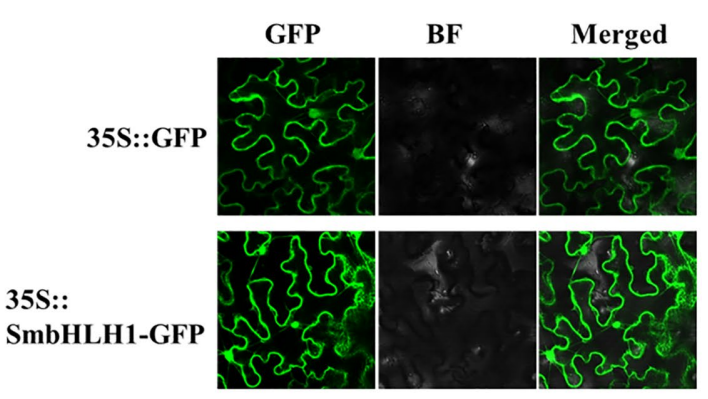

FIGURE 2 I Relationship of anthocyanin contents with the expression of SmTT8 and SmbHLH1. (A) The anthocyanin contents in the fruit and stem peels of six eggplant cultivars; (B,C) The expression levels of SmTT8 and SmbHLH1 in the fruit and stem peels of six eggplant cultivars. Bars represent means \pm SD of three biological replicates; and (D) Subcellular localization analysis of SmbHLH1.

peels were changed from purple to green, which could be accumulated anthocyanins in cultivar No.109. However, the fruit peels of cultivar No.109 was orange with little anthocyanins, no significant difference was found comparing with the two 35S::SmbHLH1 transgenic eggplant lines. Next, we evaluated the anthocyanin contents and the expression levels of anthocyanin biosynthetic genes ( $\mathrm{SmCHS}$, SmCHI, SmF3H, SmF3'5'H, SmDFR, and $S m A N S$ ) in the stems peel. Anthocyanin contents and expression levels of anthocyanin biosynthetic genes were lower in stems peel of the two $S m b H L H 1$ transgenic lines than in WT stems (Figures 3C,D).

\section{SmbHLH1 Could Not Interact With SmMYB113}

We performed yeast two-hybrid ( $\mathrm{Y} 2 \mathrm{H})$ assays to determine whether SmbHLH1 regulated anthocyanin biosynthesis through interaction with SmMYB113, which has been reported as a critical anthocyanin regulator and interacted with SmTT8 (Zhou et al., 2020). Positive $\beta$-gal activity was observed only in yeast that contained $\mathrm{AD}-\mathrm{SmMYB} 113$ plus $\mathrm{BD}-\mathrm{SmTT} 8$ grown on $-\mathrm{T} /-\mathrm{L} / \mathrm{-H} /-\mathrm{A}$ screening medium but not in yeast that contained AD plus BD-SmbHLH1, AD plus BD-SmTT8, AD-SmMYB113 plus BD, or AD-SmMYB113 plus BD-SmbHLH1 (Figure 4A). These results indicated that SmbHLH1 could not interact with SmMYB113, whereas SmTT8 could.
Bimolecular fluorescence complementation assays were performed to further characterize the interaction between SmbHLH1/SmTT8 and SmMYB113 in vivo. As shown in Figure 4B, YFP fluorescence signals appeared only when $\mathrm{YFP}_{\mathrm{C}^{-}}$ SmMYB113 and $\mathrm{YFP}_{\mathrm{N}^{-}}$SmTT8 were co-expressed. No fluorescence was detected in cells that contained combinations of $\mathrm{YFP}_{\mathrm{C}^{-}}$ SmMYB113 plus YFP $_{\mathrm{N}}-\mathrm{SmbHLH} 1$ and other empty vector controls. In addition, we found that the interaction location of SmMYB113 and SmTT8 complexes was in the nucleus. These results confirmed that SmbHLH1 could not interact with SmMYB113 in plant cells, but SmTT8 could.

\section{SmbHLH1 Is a Negative Transcription Regulator of SmDFR and SmANS}

The cis-elements in the promoter sequences of six anthocyanin biosynthetic genes ( $\mathrm{SmCHS}, \mathrm{SmCHI}, \mathrm{SmF3H}, \mathrm{SmF3} 3^{\prime} H, S m D F R$, and $S m A N S$ ) were analyzed, and E-box or G-box cis-elements recognized by bHLH family members were found in all six promoter sequences (Supplementary Figure S4). Then, we performed a dual-luciferase assay by infiltration of tobacco leaves to analyze whether SmbHLH1 can inhibit the transcription of the anthocyanin biosynthetic genes. As a result, we found that SmDFR and SmANS promoter activities were inhibited by SmbHLH1 (Figure 5A). Because SmMYB113 can bind directly to the SmDFR promoter and activate its expression 
A
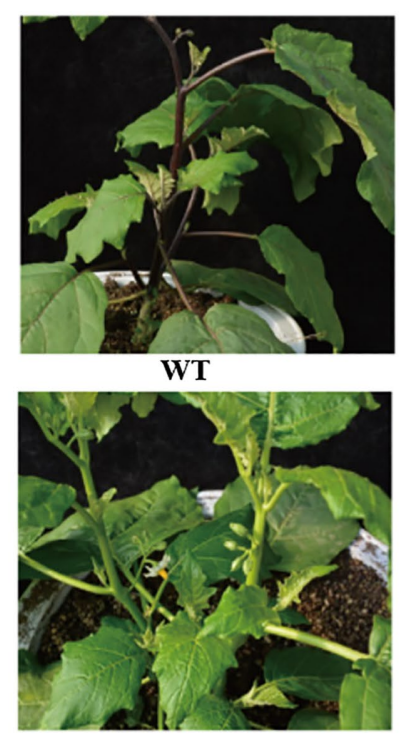

SmbHLH1-1

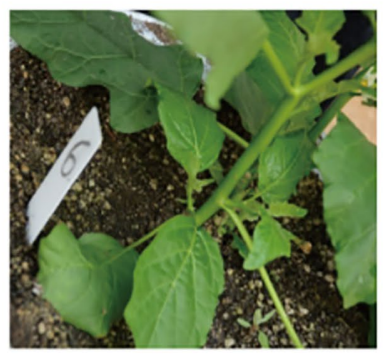

SmbHLH1-2
B

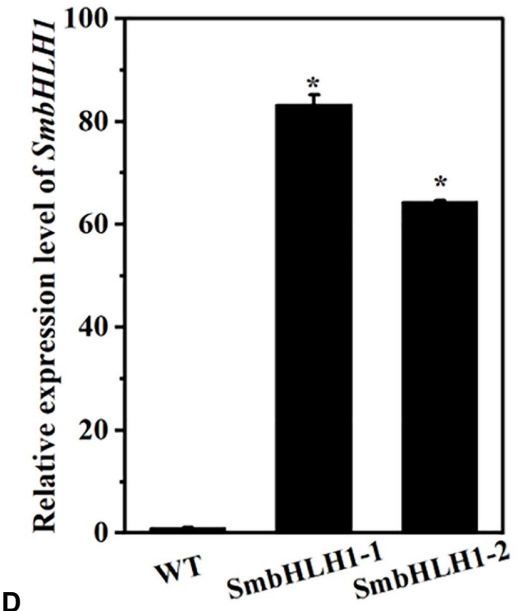

C

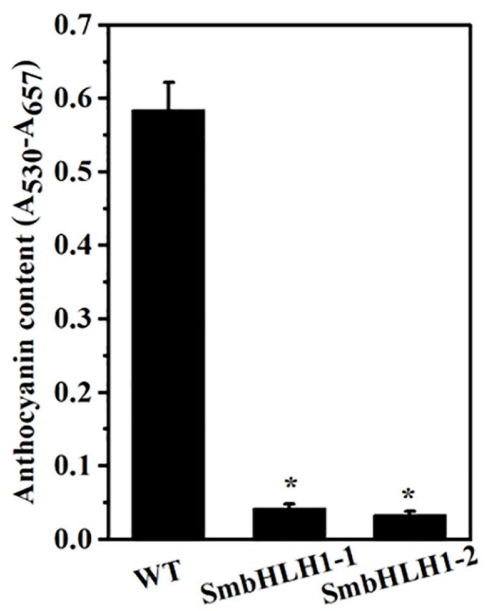

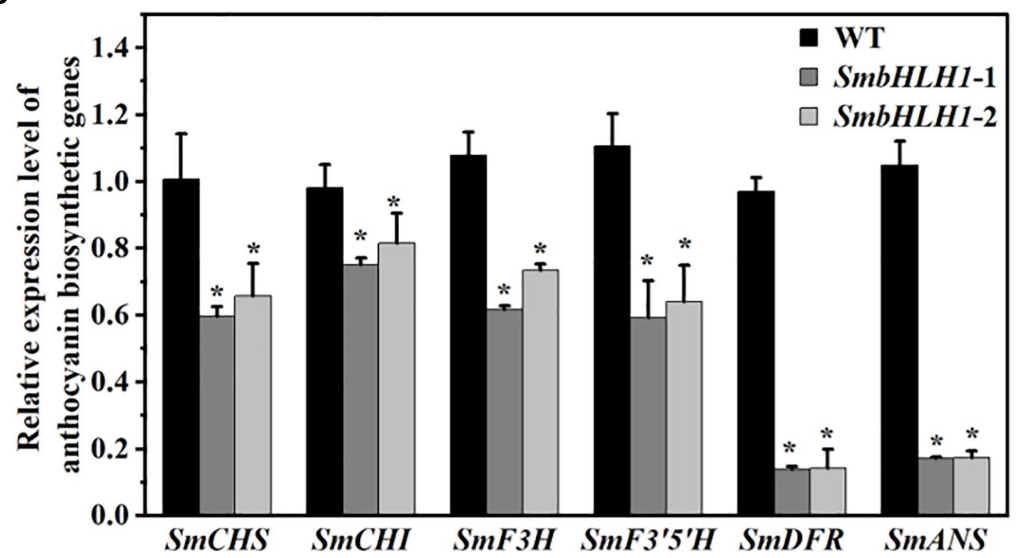

FIGURE 3 | SmbHLH1 controls anthocyanin biosynthesis in the stem peels of eggplant (cultivar No. 109). (A) The phenotypes of WT and the two SmbHLH1 transgenic lines (SmbHLH-1 and -2); (B) The relative expression level of SmbHLH1 in the two 35S::SmbHLH1 stem peels lines; (C) The anthocyanin content in the two 35S::SmbHLH1 stem peels lines; and (D) The relative expression level of anthocyanin biosynthetic genes in stem peels of the two 35S::SmbHLH1 lines. Values are means $\pm \mathrm{SD}(n=3)$. * represents significance at $p<0.05$ comparing with wild-type (WT).

(Jiang et al., 2016), SmMYB113 was co-infiltrated with SmbHLH1. As shown in Figure 5B, the inhibition of the SmDFR promoter activity by SmbHLH1 occurred in the presence of SmMYB113.

Another transient expression assay was carried out in tobacco leaves to explore the role of SmbHLH1 in anthocyanin biosynthesis. Agrobacterium tumefaciens GV3101 strains containing the empty vector pHB, SmbHLH1, SmTT8, or SmMYB113 were infiltrated or co-infiltrated into tobacco leaves. As shown in Figure 5C, pigmentation was evident after infiltration with SmMYB113 alone and co-infiltration with SmbHLH1 and SmTT8. Compared with the leaves infiltrated with $p H B$ and SmMYB113 alone, anthocyanin content decreased when SmbHLH1 was added but increased when SmTT8 was added. Next, different ratios of SmbHLH1:SmMYB113 and SmbHLH1:SmMYB113:SmTT8 were used to analyze the effect of SmbHLH1 on SmMYB113-induced anthocyanin biosynthesis (Figures 5D,E). We found that anthocyanin accumulation in infiltrated patches was negatively correlated with the dose of SmbHLH1. These results suggested that SmbHLH1 was a key negative regulator of anthocyanin accumulation in eggplant, directly inhibiting SmDFR and SmANS expression through a SmMYB113-independent pathway.

\section{Overview of RNA-Seq Analysis}

To explore how SmbHLH1 regulates gene expression at the whole-genome level, total RNA was extracted from stems of the WT and two SmbHLH1 transgenic lines for RNA-seq analysis. A total of 29,840 genes or transcripts were obtained. Subsequently, we performed pairwise comparisons of transcript abundance to identify differentially expressed genes (DEGs) between the WT and transgenic lines based on an absolute fold change value of $\left|\log _{2}{ }^{\text {ratio }}\right| \geq 0.5$ with $p<0.001$. In total, 2,120 genes were differentially expressed in SmbHLH1-1 stems compared with the WT (933 up; 1,187 down), and 3,467 genes were differentially expressed in SmbHLH1-2 stems compared with the WT $(1,811$ up; 1,656 down; Figure 6A; Supplementary Table S2). Among these DEGs, only 126 genes were upregulated and 181 genes were downregulated simultaneously in both $S m b H L H 1$-overexpressing lines compared with the WT (Figure 6B). 


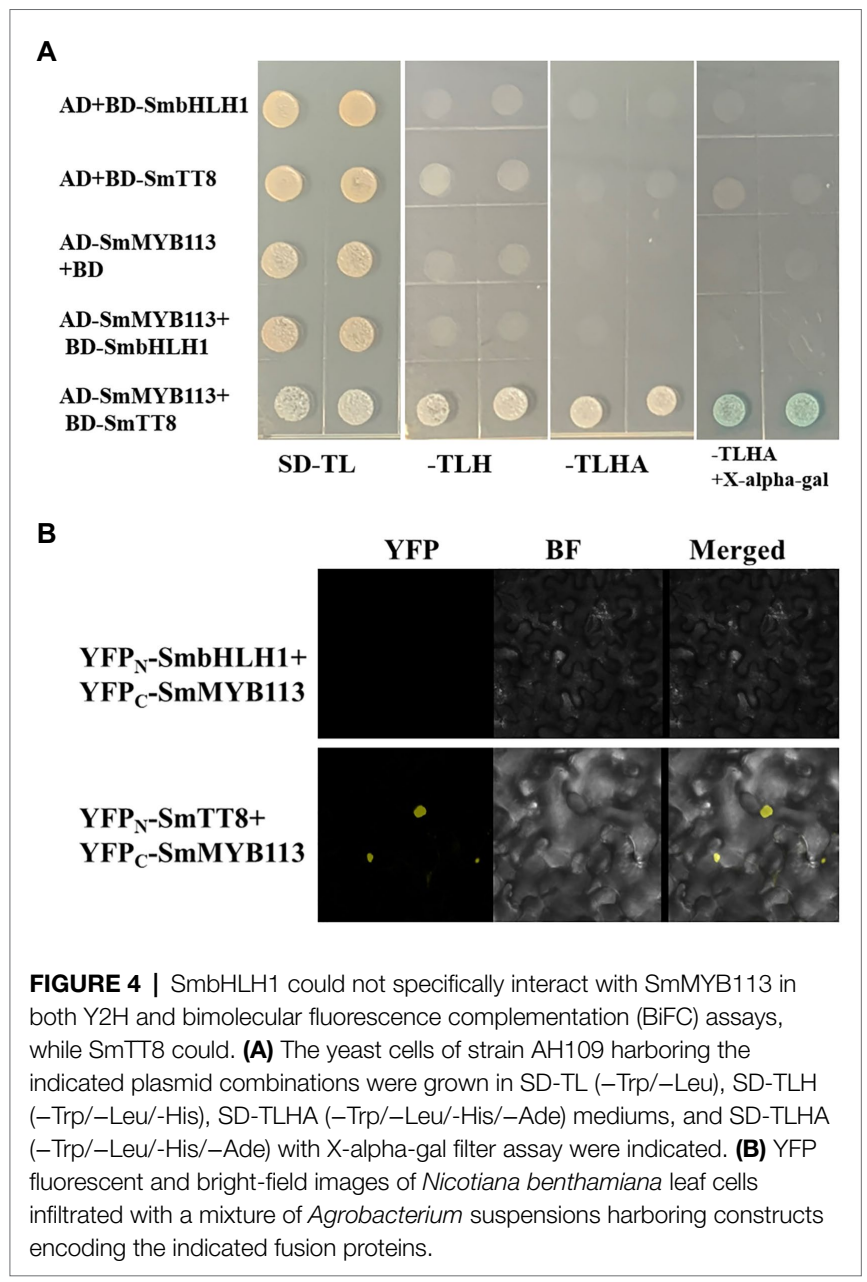

GO functional enrichment analysis was performed on the 126 upregulated and 181 downregulated genes shared by the two SmbHLH1 transgenic eggplant lines (Figures 6C,D). The 126 upregulated genes were enriched in $29 \mathrm{GO}$ terms, and the 181 downregulated genes were enriched in 27 GO terms. In the "biological process" category, "cell proliferation" was enriched only in the downregulated genes, and "carbon utilization," "reproduction," and "reproductive process" were enriched only in the upregulated genes. In the "molecular function" category, "nutrient reservoir activity" was enriched only in the upregulated genes, and "structural molecule activity" was enriched only in the downregulated genes.

The Kyoto Encyclopedia of Genes and Genomes (KEGG) pathways of the 126 shared upregulated genes and the 181 shared downregulated genes were identified using a value of $p$ less than 0.05 as the cut-off (Table 1). As shown in Table 1, the "carbon metabolism" and "starch and sucrose metabolism" pathways were enriched in the upregulated genes, and the "flavonoid biosynthesis" and "anthocyanin biosynthesis" pathways were enriched in the downregulated genes. These results suggest that SmbHLH1 inhibited anthocyanin biosynthesis but promoted carbon metabolism. We next examined glucose, fructose, and sucrose contents in stems, functional leaves, and expanding leaves. The two SmbHLH1 transgenic eggplant lines showed
TABLE 1 | Kyoto Encyclopedia of Genes and Genomes (KEGG) pathway enrichment analysis of common upregulated and downregulated genes in two SmbHLH1 transgenic eggplant stem peels lines vs. WT.

\begin{tabular}{|c|c|}
\hline Upregulated genes & Downregulated genes \\
\hline ko01100 Metabolic pathways (26) & ko01100 Metabolic pathways (31) \\
\hline $\begin{array}{l}\text { ko01110 Biosynthesis of secondary } \\
\text { metabolites (15) }\end{array}$ & $\begin{array}{l}\text { ko01110 Biosynthesis of secondary } \\
\text { metabolites (19) }\end{array}$ \\
\hline \multirow{3}{*}{$\begin{array}{l}\text { ko01200 Carbon metabolism (7) } \\
\text { ko:K00382 DLD; dihydrolipoamide } \\
\text { dehydrogenase }\end{array}$} & ko00564 Glycerophospholipid \\
\hline & metabolism (6) \\
\hline & ko04141 Protein processing in \\
\hline ko:K00873 PK; pyruvate kinase & endoplasmic reticulum (5) \\
\hline \multirow{2}{*}{$\begin{array}{l}\text { ko:K01602 rbcS; ribulose- } \\
\text { bisphosphate carboxylase small chain }\end{array}$} & ko00941 Flavonoid biosynthesis (5) \\
\hline & ko:K00475 F3H; Naringenin \\
\hline \multirow{2}{*}{$\begin{array}{l}\text { ko:K01913 AAE7; acetate/butyrate--- } \\
\text { CoA ligase }\end{array}$} & 3-dioxygenase \\
\hline & ko:K00660 CHS; Chalcone synthase \\
\hline \multirow{2}{*}{$\begin{array}{l}\text { ko:K02437 gcvH; glycine cleavage } \\
\text { system H protein }\end{array}$} & $(\mathrm{CHS})$ \\
\hline & ko:K05277 ANS; Anthocyanidin \\
\hline \multirow{2}{*}{$\begin{array}{l}\text { ko:K03841 FBP; fructose-1,6- } \\
\text { bisphosphatase I }\end{array}$} & synthase \\
\hline & ko:K13065 E2.3.1.133; Shikimate \\
\hline \multirow{2}{*}{$\begin{array}{l}\text { ko:K05605 HIBCH; } \\
\text { 3-hydroxyisobutyryl-CoA hydrolase }\end{array}$} & O-hydroxycinnamoyl transferase \\
\hline & ko:K13082 DFR; bifunctional \\
\hline $\begin{array}{l}\text { ko04075 Plant hormone signal } \\
\text { transduction (5) }\end{array}$ & $\begin{array}{l}\text { dihydroflavonol 4-reductase (DFR)/ } \\
\text { flavanone 4-reductase }\end{array}$ \\
\hline $\begin{array}{l}\text { ko04016 MAPK signaling pathway } \\
\text { - plant (5) }\end{array}$ & $\begin{array}{l}\text { ko04075 Plant hormone signal } \\
\text { transduction (4) }\end{array}$ \\
\hline ko04626 Plant-pathogen interaction (4) & ko00942 Anthocyanin biosynthesis (1) \\
\hline $\begin{array}{l}\text { ko00630 Glyoxylate and dicarboxylate } \\
\text { metabolism (4) }\end{array}$ & $\begin{array}{l}\text { ko:K12930 BZ1; Aanthocyanidin } \\
\text { 3-O-glucosyltransferase }\end{array}$ \\
\hline
\end{tabular}

ko00010 Glycolysis/Gluconeogenesis (4)

ko00500 Starch and sucrose metabolism (2)

ko:K01177 E3.2.1.2; beta-amylase

ko:K01179 E3.2.1.4; endoglucanase

significant increases in glucose, fructose, and sucrose content compared with the WT (Supplementary Figure S5). These results are consistent with the transcriptomic analysis.

\section{DISCUSSION}

The bHLH, R2R3-MYB, and WD repeat proteins can form a ternary MBW complex to modulate the expression of anthocyanin biosynthesis-related genes. Previous studies have revealed that several bHLH TFs, including AtTT8, AtGL3, AtEGL3, PhAN1, PhJAF13, GhMYC1, SlAN1, MdbHLH3, and PbbHLH2, interact with their respective MYB partners AtPAP1, AtTT2, PhAN2, GhMYB10, SlAN2, MdMYB1, and PbMYB9/10/10b to regulate anthocyanin or proanthocyanidin biosynthesis in Arabidopsis, Petunia hybrida, Gerbera hybrida, S. lycopersicum, M. domestica, and Pyrus bretschneideri (Elomaa et al., 2003; Koes et al., 2005; Ramsay and Glover, 2005; Xie et al., 2012; Sun et al., 2020a; Li et al., 2021b). Xie et al. (2012) reported that two regions (amino acids 1-24 and 186-228) at the $N$ terminus of MdbHLH3 were crucial for the interaction between MdbHLH3 and 
A

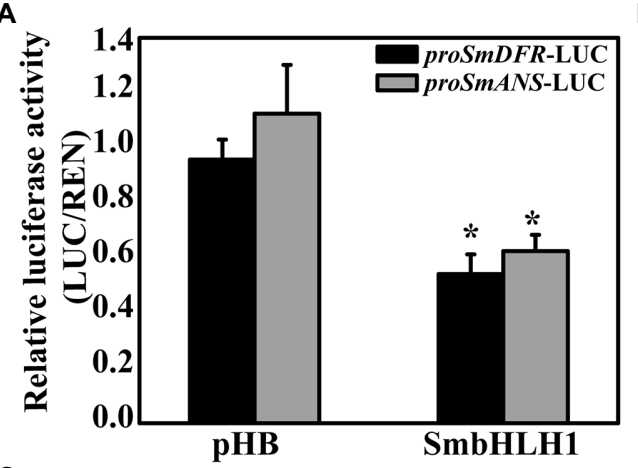

C

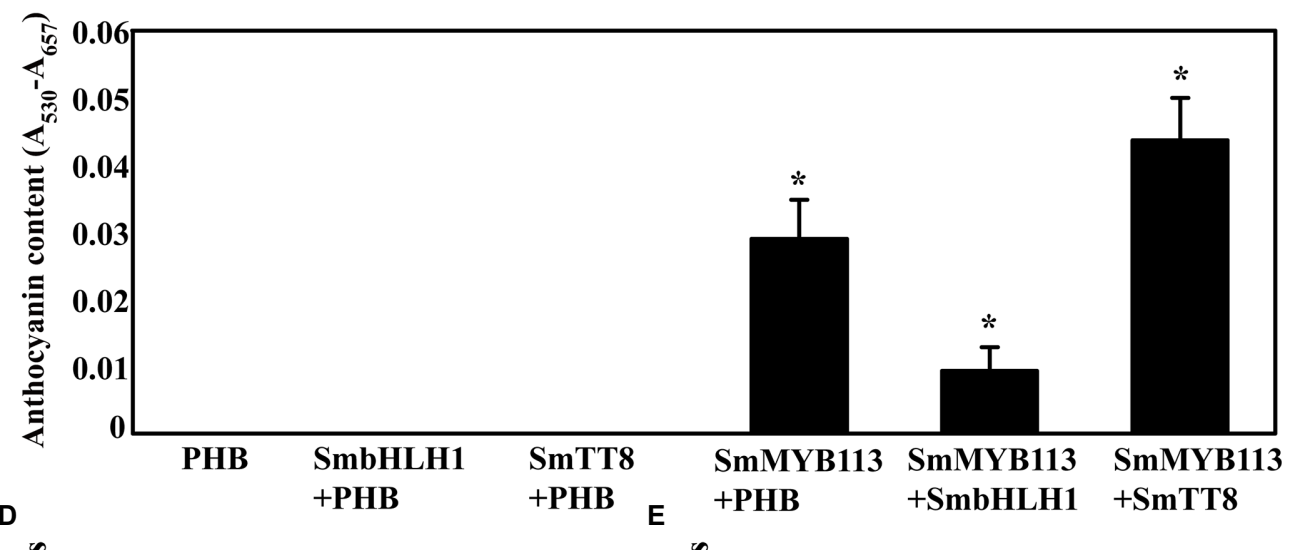

B

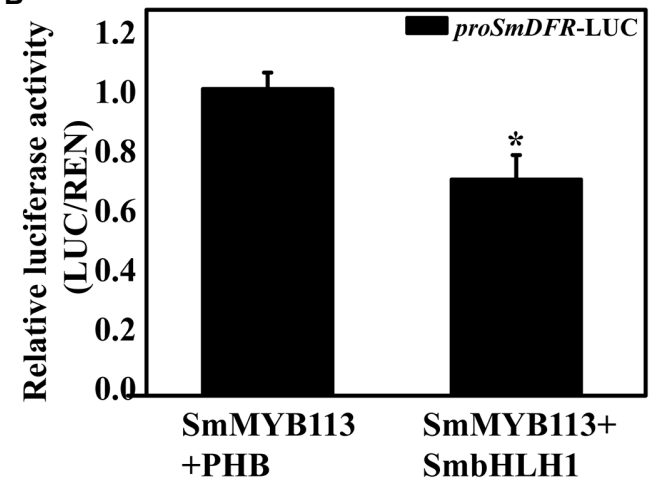

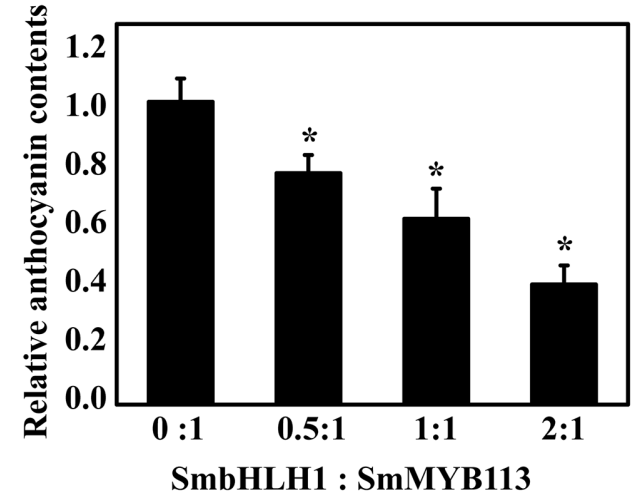

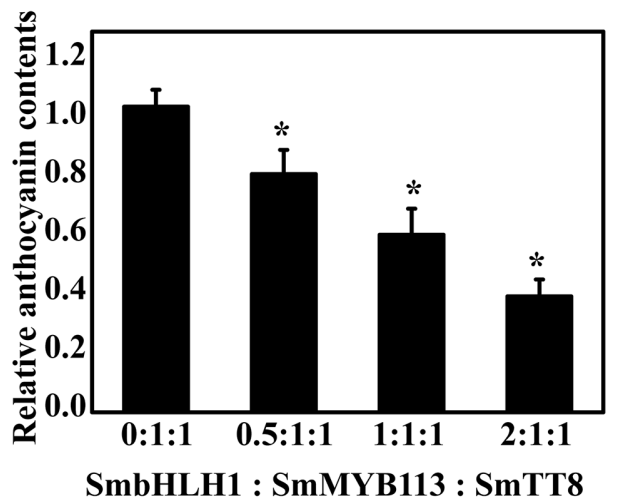

FIGURE 5 | Dual-luciferase and transient analysis of SmbHLH1 on anthocyanin accumulation. (A) Inhibition of the activity of the SmDFR and SmANS gene promoters by SmbHLH1 in transient expression assay in N. benthamiana leaves; (B) SmbHLH1 decreased the ratio of LUC/REN of the SmDFR promoter activated by SmMYB113; and (C) SmbHLH1 inhibited SmMYB113-induced anthocyanin accumulation while SmTT8 enhanced in $N$. benthamiana leaves. (D,E) The relative anthocyanin contents induced by different proportions of SmbHLH1:SmMYB113 and SmbHLH1:SmMYB113:SmTT8 in infiltrated regions. *Represents significance at $p<0.05$ comparing with the control.

MdMYB1. In this study, we found that SmbHLH1, SmTT8, and MdbHLH3 were clustered into one clade by phylogenetic analysis (Supplementary Figure S3), and the $N$ terminus (amino acids 1-158 and 188-193) and C terminus (amino acids 633-659) of SmbHLH1 differed significantly from those of SmTT8 (Figure 1). SmbHLH1 did not interact with SmMYB113 in both $\mathrm{Y} 2 \mathrm{H}$ and BiFC assays (Figure 4), whereas SmTT8 did which was corresponding to the results reported by Moglia et al. (2020). These results suggested that the N-terminal portion of SmTT8 was crucial for its interaction with SmMYB113. Simultaneously, the pattern of SmbHLH1 expression contrasted with those of SmTT8 expression and anthocyanin accumulation (Figure 2). Previous studies have reported that the enhancement of anthocyanin biosynthesis by SmTT8 is dependent on SmMYB113 (Moglia et al., 2020; Zhou et al., 2020). However, we found that over-expression of SmbHLH1 inhibited anthocyanin accumulation (Figure 3). Dual-luciferase assays revealed that SmbHLH1 directly repressed the expression of SmDFR and SmANS (Figure 5). Because the color of cultivar No.109 tissues was different, the phenotype changes of $35 S:: S m b H L H 1$ transgenic eggplant lines were different. For the tissues with anthocyanins accumulation were changed from purple to green, such as 


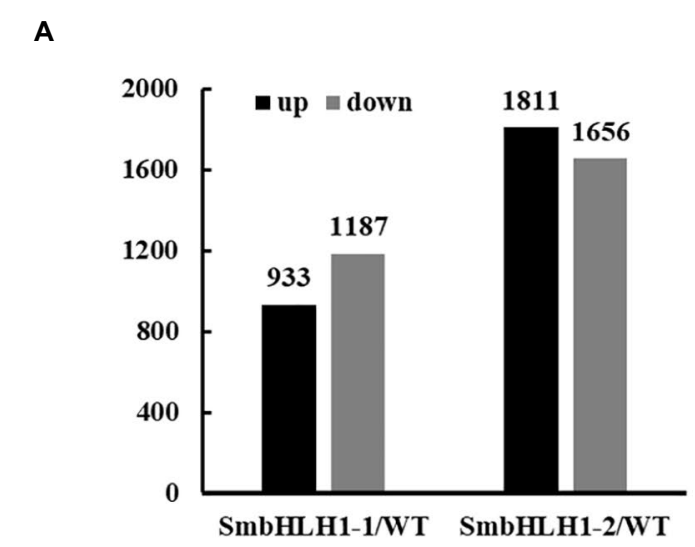

C

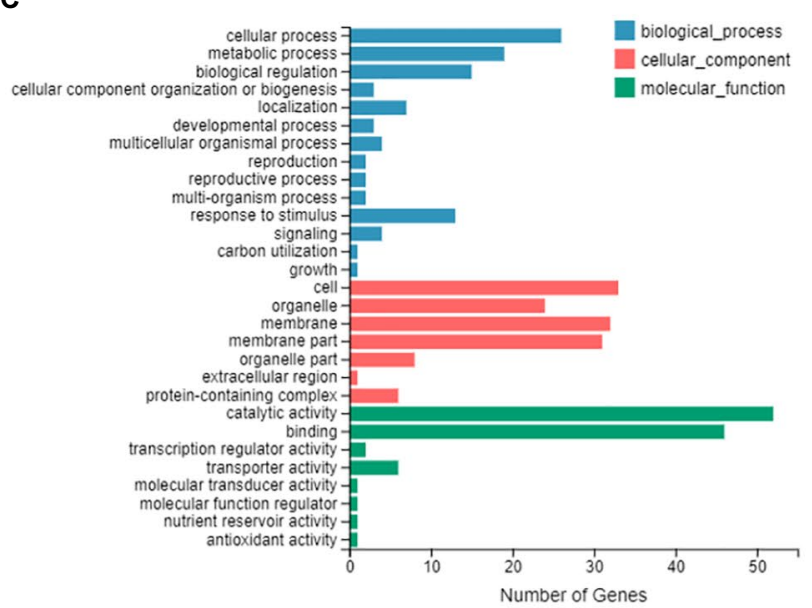

B

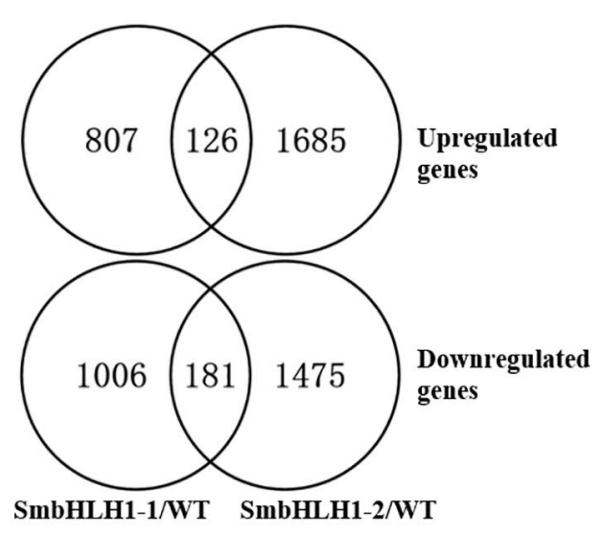

D

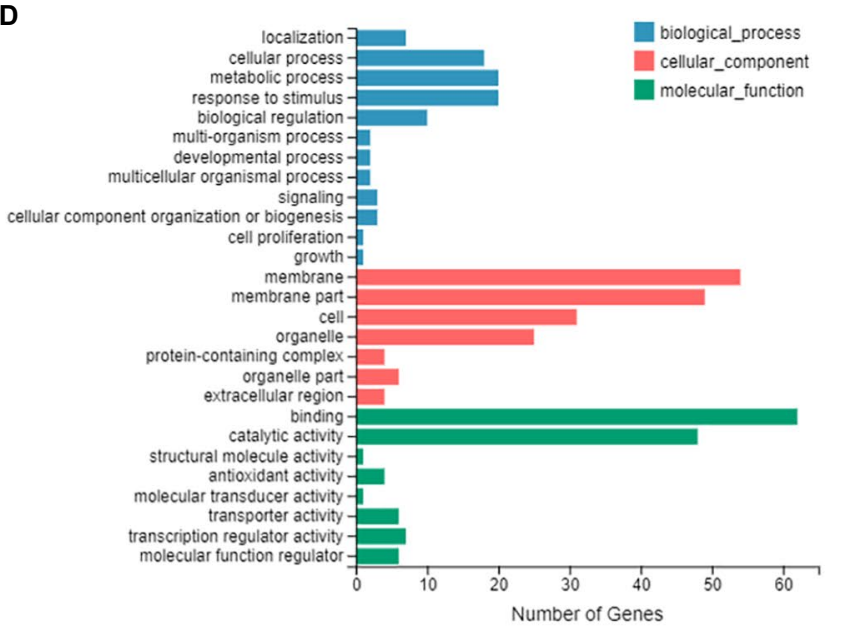

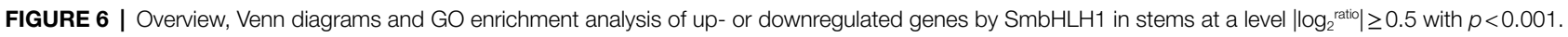
(A) Number of upregulated and downregulated genes in the two 35S::SmbHLH1 stem peels lines vs. WT with RNA-seq. (B) Schematic of the Venn diagram analysis of common upregulated and downregulated genes [differentially expressed genes (DEGs)] in the two 35S::SmbHLH1 stem peels lines vs. WT. (C,D) GO functional enrichment analysis of common upregulated (C) and downregulated (D) genes.

stalks, sepals, and stem peels. However, no significant color change was found in the tissues with little anthocyanins content, such as the fruit peels. Taken all above, we could conclude that $S m b H L H 1$ was a repressor in anthocyanin biosynthesis. Moreover, the silencing of $S m b H L H 1$ in fruit peel tissue to verify the fruit can accumulate more anthocyanins is worthy in the future,

BHLH proteins are the second largest class of transcription factors in plants and can be divided into 26-32 subgroups (Carretero-Paulet et al., 2010; Pires and Dolan, 2010); they play important roles in stress tolerance, reproduction, and secondary metabolite biosynthesis (Sun et al., 2020b; Guo et al., 2021). It is worth noting that AtTT8, AtGL3, AtEGL1, and AtMYC1, which are positive regulator of anthocyanin biosynthesis, were placed into subgroup IIIf (Heim et al., 2003). However, AtMYC1 orthologs in maize and C. praecox, as well as another bHLH TF from subgroup IVd, have been reported to negatively regulate anthocyanin biosynthesis (Burr et al., 1996; Zhao et al., 2018). Here, SmTT8 and SmbHLH1 are both eggplant orthologs of AtTT8, but they played opposite roles in anthocyanin biosynthesis. It is not uncommon for such homologous genes to exhibit different functions in the plant. For example, Li et al. (2021a) reported that the biological function of MS1 in pollen development differed in the evolution of Poaceae plants. The different biological functions of homologous genes probably result from differences in some amino acids. Taking all of our results into consideration, we speculate that the N-terminal front portion of SmbHLH1 may be the key to its role in anthocyanin biosynthesis.

RNA-seq analysis revealed that overexpression of $\mathrm{SmbHLH1}$ reduced the expression of anthocyanin biosynthetic genes while enhancing that of genes involved in carbon metabolism and starch and sucrose metabolism. Combined with another phenotype of the two SmbHLH1 transgenic eggplant lines, which were greener than the WT due to increased chlorophyll content (Figure 3; Supplementary Figure S4), we speculated that more light was absorbed, resulting in significant increases in glucose, fructose, and sucrose content. Anthocyanin and chlorophyll are two major plant pigments. Anthocyanin can protect plants from UV light and oxidative damage, whereas 
chlorophyll is essential for the conversion of light energy into stored chemical energy. Previous studies have reported that anthocyanin often accumulates in young leaves but degrades in mature leaves, which is beneficial for the gradual accumulation of chlorophyll in many plants (Oren-Shamir, 2009; Ren et al., 2019). On this basis, we speculate that the increased chlorophyll content may be directly regulated by $S m b H L H 1$ or caused by a decrease in anthocyanin, suggesting the existence of a much more complex molecular mechanism that is worthy of study in the future.

\section{CONCLUSION}

In the eggplant genome, we found that both SmbHLH1 and SmTT8 are homologs of TT8. Their amino acid sequences differed only at the $\mathrm{N}$ and $\mathrm{C}$ termini, but they had contrasting functions in anthocyanin biosynthesis. SmbHLH1 repressed the expression of SmDFR and SmANS and inhibited anthocyanin biosynthesis through SmMYB113-independent pathway. We identified SmbHLH1 as a new target to silence to produce more anthocyanins in eggplant fruit peel. In addition, RNA-seq analysis revealed that genes involved in carbon metabolism and starch and sucrose metabolism were upregulated by overexpression of $\mathrm{SmbHLH1}$ in eggplant.

\section{DATA AVAILABILITY STATEMENT}

The datasets presented in this study can be found in online repositories. The names of the repository/repositories and accession number(s) can be found at: NCBI BioProject, PRJNA761256.

\section{REFERENCES}

Allan, A. C., Hellens, R. P., and Laing, W. A. (2008). MYB transcription factors that colour our fruit. Trends Plant Sci. 13, 99-102. doi: 10.1016/j. tplants.2007.11.012

An, X.-H., Tian, Y., Chen, K.-Q., Liu, X.-J., Liu, D.-D., Xie, X.-B., et al. (2014). MdMYB9 and MdMYB11 are involved in the regulation of the ja-induced biosynthesis of anthocyanin and proanthocyanidin in apples. Plant Cell Physiol. 56, 650-662. doi: 10.1093/pcp/pcu205

Atchley, W. R., Terhalle, W., and Dress, A. (1999). Positional dependence, cliques, and predictive motifs in the bHLH protein domain. J. Mol. Evol. 48, 501-516. doi: 10.1007/PL00006494

Barchi, L., Pietrella, M., Venturini, L., Minio, A., Toppino, L., Acquadro, A., et al. (2019). A chromosome-anchored eggplant genome sequence reveals key events in Solanaceae evolution. Sci. Rep. 9:11769. doi: 10.1038/s41598-019-47985-w

Baudry, A., Caboche, M., and Lepiniec, L. (2006). TT8 controls its own expression in a feedback regulation involving TTG1 and homologous MYB and bHLH factors, allowing a strong and cell-specific accumulation of flavonoids in Arabidopsis thaliana. Plant J. 46, 768-779. doi: 10.1111/j.1365313X.2006.02733.x

Burr, F. A., Burr, B., Scheffler, B. E., Blewitt, M., Wienand, U., and Matz, E. C. (1996). The maize repressor-like gene intensifierl shares homology with the r1/b1 multigene family of transcription factors and exhibits missplicing. Plant Cell 8:1249. doi: 10.1105/tpc.8.8.1249

Carretero-Paulet, L., Galstyan, A., Roig-Villanova, I., Martínez-García, J. F., Bilbao-Castro, J. R., and Robertson, D. L. (2010). Genome-wide classification

\section{AUTHOR CONTRIBUTIONS}

FY, JL, and MW designed the research. ZD, ST, JL, and GY performed experiments. JL and ZD analyzed data. JL wrote the manuscript. FY and JL read and modified the manuscript. All authors contributed to the article and approved the submitted version.

\section{FUNDING}

This work was supported by the National Natural Science Foundation of China (grant number: 31672169), Youth Project of Shandong Provincial Natural Science Foundation (grant number: ZR201911120670), Key Project of Shandong Provincial Natural Science Foundation (ZR2020KC039), and Special Project Protected Horticulture Advantage Team of Shandong Agricultural University 'Double First-Class' Science and Technology Team (grant number: SYL2017YSTD07).

\section{ACKNOWLEDGMENTS}

We are very grateful for the reviewers for their comments and suggestions, which is helpful for improving the quality of our manuscript.

\section{SUPPLEMENTARY MATERIAL}

The Supplementary Material for this article can be found online at: https://www.frontiersin.org/articles/10.3389/fpls.2021.757936/ full\#supplementary-material

and evolutionary analysis of the bHLH family of transcription factors in Arabidopsis, poplar, rice, moss, and algae. Plant Physiol. 153, 1398-1412. doi: 10.1104/pp.110.153593

Elomaa, P., Uimari, A., Mehto, M., Albert, V. A., Laitinen, R. A., and Teeri, T. H. (2003). Activation of anthocyanin biosynthesis in Gerbera hybrida (Asteraceae) suggests conserved protein-protein and protein-promoter interactions between the anciently diverged monocots and eudicots. Plant Physiol. 133, 1831-1842. doi: 10.1104/pp.103.026039

Guo, J., Sun, B., He, H., Zhang, Y., Tian, H., and Wang, B. (2021). Current understanding of bHLH transcription factors in plant abiotic stress tolerance. Int. J. Mol. Sci. 22:4921. doi: 10.3390/ijms22094921

He, Q., Jones, D. C., Li, W., Xie, F., Ma, J., Sun, R., et al. (2016). Genomewide identification of R2R3-MYB genes and expression analyses during abiotic stress in Gossypium raimondii. Sci. Rep. 6:22980. doi: 10.1038/ srep 22980

Heim, M. A., Jakoby, M., Werber, M., Martin, C., Weisshaar, B., and Bailey, P. C. (2003). The basic helix-loop-helix transcription factor family in plants: a genome-wide study of protein structure and functional diversity. Mol. Biol. Evol. 20, 735-747. doi: 10.1093/molbev/msg088

Hirakawa, H., Shirasawa, K., Miyatake, K., Nunome, T., Negoro, S., Ohyama, A., et al. (2014). Draft genome sequence of eggplant (Solanum melongena L.): the representative solanum species indigenous to the old world. DNA Res. 21, 649-660. doi: 10.1093/dnares/dsu027

Hu, D. G., Yu, J. Q., Han, P. L., Xie, X. B., Sun, C. H., Zhang, Q. Y., et al. (2019). The regulatory module MdPUB29-MdbHLH3 connects ethylene biosynthesis with fruit quality in apple. New Phytol. 221, 1966-1982. doi: $10.1111 /$ nph.15511 
Huang, J., Guo, Y., Sun, Q., Zeng, W., Li, J., Li, X., et al. (2018). Genome-wide identification of R2R3-MYB transcription factors regulating secondary cell wall thickening in cotton fiber development. Plant Cell Physiol. 60, 687-701. doi: $10.1093 / \mathrm{pcp} / \mathrm{pcy} 238$

Ilk, N., Ding, J., Ihnatowicz, A., Koornneef, M., and Reymond, M. (2015). Natural variation for anthocyanin accumulation under high-light and low-temperature stress is attributable to the ENHANCER OF AG-4 2 (HUA2) locus in combination with production of anthocyanin pigmenT1 (PAP1) and PAP2. New Phytol. 206, 422-435. doi: 10.1111/ nph.13177

Jia, N., Wang, J. J., Liu, J., Jiang, J., Sun, J., Yan, P., et al. (2021). DcTT8, a bHLH transcription factor, regulates anthocyanin biosynthesis in Dendrobium candidum. Plant Physiol. Biochem. 162, 603-612. doi: 10.1016/j. plaphy.2021.03.006

Jiang, M. M., Ren, L., Lian, H. L., Liu, Y., and Chen, H. Y. (2016). Novel insight into the mechanism underlying light-controlled anthocyanin accumulation in eggplant (Solanum melongena L.). Plant Sci. 249, 46-58. doi: $10.1016 /$ j.plantsci.2016.04.001

Koes, R., Verweij, W., and Quattrocchio, F. (2005). Flavonoids: a colorful model for the regulation and evolution of biochemical pathways. Trends Plant Sci. 10, 236-242. doi: 10.1016/j.tplants.2005.03.002

Konczak, I., and Zhang, W. (2004). Anthocyanins-more than nature's colours. J. Biomed. Biotechnol. 2004, 239-240. doi: 10.1155/S1110724304407013

Kumar, S., Stecher, G., and Tamura, K. (2016). MEGA7: molecular evolutionary genetics analysis version 7.0 for bigger datasets. Mol. Biol. Evol. 33, 18701874. doi: $10.1093 / \mathrm{molbev} / \mathrm{msw} 054$

Li, P., Chen, B., Zhang, G., Chen, L., Dong, Q., Wen, J., et al. (2016b). Regulation of anthocyanin and proanthocyanidin biosynthesis by Medicago truncatula bHLH transcription factor MtTT8. New Phytol. 210, 905-921. doi: 10.1111/ nph.13816

Li, J., Gao, Z., Zhou, L., Li, L. Z., Zhang, J. H., Liu, Y., et al. (2019). Comparative transcriptome analysis reveals $\mathrm{K}^{+}$transporter gene contributing to salt tolerance in eggplant. BMC Plant Biol. 19:67. doi: 10.1186/s12870-019-1663-8

Li, J., He, Y. J., Zhou, L., Liu, Y., Jiang, M. M., Ren, L., et al. (2018). Transcriptome profiling of genes related to light-induced anthocyanin biosynthesis in eggplant (Solanum melongena L.) before purple color becomes evident. BMC Genomics 19:324. doi: 10.1186/S12864-018-4693-Y

Li, J., Jiang, M. M., Ren, L., Liu, Y., and Chen, H. Y. (2016a). Identification and characterization of CBL and CIPK gene families in eggplant (Solanum melongena L.). Mol. Gen. Genomics. 291, 1769-1781. doi: 10.1007/ s00438-016-1218-8

Li, J., Ren, L., Gao, Z., Jiang, M., Liu, Y., Zhou, L., et al. (2017). Combined transcriptomic and proteomic analysis constructs a new model for lightinduced anthocyanin biosynthesis in eggplant (Solanum melongena L.). Plant Cell Environ. 40, 3069-3087. doi: 10.1111/pce.13074

Li, J., Wang, Z., Chang, Z., He, H., Tang, X., Ma, L., et al. (2021a). A functional characterization of TaMs1 orthologs in Poaceae plants. Crop J. doi: 10.1016/j. cj.2020.12.002 (in press).

Li, X., Xiang, F., Han, W., Qie, B., Zhai, R., Yang, C., et al. (2021b). The MIR-domain of PbbHLH2 is involved in regulation of the anthocyanin biosynthetic pathway in "red zaosu" (pyrusbretschneideri rehd.) pear fruit. Int. J. Mol. Sci. 22:3026. doi: 10.3390/ijms22063026

Li, Q., Zhang, C., Li, J., Wang, L., and Ren, Z. (2012). Genome-wide identification and characterization of R2R3MYB family in Cucumis sativus. PLoS One 7:e47576. doi: 10.1371/journal.pone.0047576

Livak, K. J., and Schmittgen, T. D. (2001). Analysis of relative gene expression data using real-time quantitative PCR and the 2(-Delta Delta $\mathrm{C}(\mathrm{T})$ ) method. Methods 25, 402-408. doi: 10.1006/meth.2001.1262

Massari, M. E., and Murre, C. (2000). Helix-loop-helix proteins: regulators of transcription in eucaryotic organisms. Mol. Cell. Biol. 20, 429-440. doi: 10.1128/MCB.20.2.429-440.2000

Moglia, A., Florio, F. E., Iacopino, S., Guerrieri, A., Milani, A. M., Comino, C., et al. (2020). Identification of a new R3 MYB type repressor and functional characterization of the members of the MBW transcriptional complex involved in anthocyanin biosynthesis in eggplant (S. melongena L.). PLoS One 15:e0232986. doi: 10.1371/journal.pone.0232986

Mol, J., Grotewold, E., and Koes, R. (1998). How genes paint flowers and seeds. Trends Plant Sci. 3, 212-217. doi: 10.1016/S1360-1385(98) $01242-4$
Montefiori, M., Brendolise, C., Dare, A. P., Lin-Wang, K., Davies, K. M., Hellens, R. P., et al. (2015). In the Solanaceae, a hierarchy of bHLHs confer distinct target specificity to the anthocyanin regulatory complex. J. Exp. Bot. 66, 1427-1436. doi: 10.1093/jxb/eru494

Neff, M. M., and Chory, J. (1998). Genetic interactions between phytochrome A, phytochrome B, and cryptochrome 1 during Arabidopsis development. Plant Physiol. 118, 27-35. doi: 10.1104/pp.118.1.27

Noda, Y., Kneyuki, T., Igarashi, K., Mori, A., and Packer, L. (2000). Antioxidant activity of nasunin, an anthocyanin in eggplant peels. Toxicology 148, 119-123. doi: 10.1016/s0300-483x(00)00202-x

Oren-Shamir, M. (2009). Does anthocyanin degradation play a significant role in determining pigment concentration in plants? Plant Sci. 177, 310-316. doi: 10.1152/japplphysiol.00626.2005

Pires, N., and Dolan, L. (2010). Origin and diversification of basic-helix-loophelix proteins in plants. Mol. Biol. Evol. 27, 862-874. doi: 10.1093/molbev/ msp288

Ramsay, N. A., and Glover, B. J. (2005). MYB-bHLH-WD40 protein complex and the evolution of cellular diversity. Trends Plant Sci. 10, 63-70. doi: 10.1016/j.tplants.2004.12.011

Ren, J., Liu, Z., Chen, W., Xu, H., and Feng, H. (2019). Anthocyanin degrading and chlorophyll accumulation lead to the formation of bicolor leaf in ornamental kale. Int. J. Mol. Sci. 20:603. doi: 10.3390/ijms20030603

Shi, S. L., Liu, Y., He, Y. J., Li, L. Z., Li, D. L., and Chen, H. Y. (2021). R2R3-MYB transcription factor SmMYB75 promotes anthocyanin biosynthesis in eggplant (Solanum melongena L.). Sci. Hortic. 282:110020. doi: 10.1016/j. scienta.2021.110020

Song, S., Qi, T., Fan, M., Zhang, X., Gao, H., Huang, H., et al. (2013). The bHLH subgroup IIId factors negatively regulate jasmonate-mediated plan defense and development. PLoS Genet. 9:e1003653. doi: 10.1371/journal. pgen. 1003653

Sparkes, I. A., Runions, J., Kearns, A., and Hawes, C. (2006). Rapid, transient expression of fluorescent fusion proteins in tobacco plants and generation of stably transformed plants. Nat. Protoc. 1, 2019-2025. doi: 10.1038/ nprot.2006.286

Sun, C., Deng, L., Du, M., Zhao, J., Chen, Q., Huang, T., et al. (2020a). A transcriptional network promotes anthocyanin biosynthesis in tomato flesh. Mol. Plant 13, 42-58. doi: 10.1016/j.molp.2019.10.010

Sun, W., Jin, X., Ma, Z., Chen, H., and Liu, M. (2020b). Basic helix-loophelix (bHLH) gene family in Tartary buckwheat (Fagopyrum tataricum): genome-wide identification, phylogeny, evolutionary expansion and expression analyses. Int. J. Biol. Macromol. 155, 1478-1490. doi: 10.1016/j. ijbiomac.2019.11.126

Tohge, T., de Souza, L. P., and Fernie, A. R. (2017). Current understanding of the pathways of flavonoid biosynthesis in model and crop plants. J. Exp. Bot. 68, 4013-4028. doi: 10.1093/jxb/erx177

Tsuda, T. (2012). Dietary anthocyanin-rich plants: biochemical basis and recent progress in health benefits studies. Mol. Nutr. Food Res. 56, 159-170. doi: $10.1002 / \mathrm{mnfr} .201100526$

Vasudevan, S., Ponraj, K., Unni, S. C., Joy, N., and Radhakrishnan, E. K. (2005). Transformation and regeneration of Solanum melongena using Agrobacterium tumefaciens. J. Plant Biol. 32, 29-32. doi: 10.1007/BF00232129

Wang, P., Su, L., Gao, H., Jiang, X., Wu, X., Li, Y., et al. (2018). Genome-wide characterization of bHLH genes in grape and analysis of their potential relevance to abiotic stress tolerance and secondary metabolite biosynthesis. Front. Plant Sci. 9:64. doi: 10.3389/fpls.2018.00064

Wang, L., Tang, W., Hu, Y., Zhang, Y., Sun, J., Guo, X., et al. (2019). A MYB/ bHLH complex regulates tissue-specific anthocyanin biosynthesis in the inner pericarp of red-centered kiwifruit Actinidia chinensis cv. Hongyang. Plant J. 99, 359-378. doi: 10.1111/tpj.14330

Xie, X. B., Li, S., Zhang, R. F., Zhao, J., Chen, Y. C., Zhao, Q., et al. (2012). The bHLH transcription factor MdbHLH3 promotes anthocyanin accumulation and fruit colouration in response to low temperature in apples. Plant Cell Environ. 35, 1884-1897. doi: 10.1111/j.1365-3040.2012.02523.x

Zhai, Y., Yu, K., Cai, S., Hu, L., Amoo, O., Xu, L., et al. (2020). Targeted mutagenesis of BnTT8 homologs controls yellow seed coat development for effective oil production in Brassica napus L. Plant Biotechnol. J. 18, 11531168. doi: $10.1111 / \mathrm{pbi} .13281$

Zhao, P., Li, X., Jia, J., Yuan, G., Chen, S., Qi, D., et al. (2018). bHLH92 from sheepgrass acts as a negative regulator of anthocyanin/proanthocyandin 
accumulation and influences seed dormancy. J. Exp. Bot. 70, 269-284. doi: $10.1093 /$ jxb/ery335

Zhao, R., Song, X. X., Yang, N., Chen, L. Q., Xiang, L., Liu, X. Q., et al. (2020). Expression of the subgroup IIIf bHLH transcription factor CpbHLH1 from Chimonanthus praecox (L.) in transgenic model plants inhibits anthocyanin accumulation. Plant Cell Rep. 39, 891-907. doi: 10.1007/s00299-020-02537-9

Zhou, L., He, Y., Li, J., Liu, Y., and Chen, H. (2020). CBFs function in anthocyanin biosynthesis by interacting with MYB113 in eggplant (Solanum melongena L.). Plant Cell Physiol. 61, 416-426. doi: 10.1093/pcp/pcz209

Conflict of Interest: The authors declare that the research was conducted in the absence of any commercial or financial relationships that could be construed as a potential conflict of interest.
Publisher's Note: All claims expressed in this article are solely those of the authors and do not necessarily represent those of their affiliated organizations, or those of the publisher, the editors and the reviewers. Any product that may be evaluated in this article, or claim that may be made by its manufacturer, is not guaranteed or endorsed by the publisher.

Copyright (c) 2021 Duan, Tian, Yang, Wei, Li and Yang. This is an open-access article distributed under the terms of the Creative Commons Attribution License (CC BY). The use, distribution or reproduction in other forums is permitted, provided the original author(s) and the copyright owner(s) are credited and that the original publication in this journal is cited, in accordance with accepted academic practice No use, distribution or reproduction is permitted which does not comply with these terms. 